\title{
LDHs生物医学复合材料的制备及其在药物输送 和诊疗方面的应用
}

\author{
梅旭安, 彭刘琪, 梁瑞政*, 卫敏* \\ 化工资源有效利用国家重点实验室, 北京化工大学, 北京 100029 \\ *通讯作者, E-mail: liangruizheng2000@163.com; weimin@mail.buct.edu.cn \\ 收稿日期: 2016-12-13; 接受日期: 2017-03-03; 网络版发表日期: 2017-03-30 \\ 国家自然科学基金(编号：21601010)资助项目
}

\begin{abstract}
摘要生命科学是关乎人类健康的重要研究领域. 基于无机纳米材料为载体的生物无机材料推动了生命 科学的发展. 水滑石(LDHs)作为一种二维无机纳米材料, 具有层板金属离子种类和比例可调控以及层间可插 层的特性, 近年来在生物医学领域受到广泛关注. 本文重点介绍了水滑石纳米复合材料的物理化学结构、作 为基因/药物载体的应用, 以及在新型诊疗一体化复合材料领域的应用和优势. 此外, 通过细胞和活体实验, 进一步探讨了水滑石纳米复合材料的细胞输运机制、诊断成像以及肿瘤治疗效果, 并展望了水滑石纳米复 合材料在生物医学领域的发展前景.
\end{abstract}

关键词水滑石 (LDHs), 插层组装, 成像治疗, 药物输送, 生物无机纳米材料

\section{1 引言}

在材料化学领域中, “插层”代表了一个化学过程. 在此过程中, 客体分子可逆地进入二维无机主体层间, 形成新型复合材料 ${ }^{[1 \sim 4]}$. 客体分子包括原子、离子、 分子和金属簇. 主体材料有自然界黏土材料和人工合 成材料, 如水滑石(LDHs)、过渡金属氧化物、硫化物 和碳材料 ${ }^{[5-9]}$.

1999年Choy课题组 ${ }^{[10]}$ 提出插层化学领域“生物无 机纳米异质材料” 的概念, 这不仅引起插层结构纳米 工程和纳米医学领域研究方式的转变, 而且使新的多 学科交叉科学以及多功能纳米异质材料体系方面有 巨大的进展 ${ }^{[1116]} .10$ 多年来, 通过插层反应, 许多具有 生物功能的客体分子插层进入 LDHs层间, 从而在药
物输送、治疗、诊断等领域都有创新性的突破, 这对 于二维生物无机纳米异质材料体系的发展具有重要 意义 ${ }^{[17 \sim 21]}$.

LDHs 的化学式通常可以表示为 $\left[\mathrm{M}^{2+}{ }_{1-x} \mathrm{M}^{3+}{ }^{-}{ }^{-}\right.$ $\left.(\mathrm{OH})_{2}\right]^{x+}\left(\mathrm{A}^{m-}\right)_{x / m} \cdot n \mathrm{H}_{2} \mathrm{O}$, 其中 $\mathrm{M}^{2+}$ 是二价金属阳离子, $\mathrm{M}^{3+}$ 是三价金属阳离子, $\mathrm{A}^{m-}$ 是层间阴离子 ${ }^{[22-25]}$. 因其化学 和晶体结构与天然矿物水滑石的化学和晶体结构非 常相似, LDHs也被称为阴离子黏土.

在医学应用上, LDHs最早是用于抗酸剂或抗胃 蛋白酶剂的药物活性成分 ${ }^{[26-28]}$. 服用含有 LDHs的抗 胃酸药物, 能够显著提高胃部的pH来缓解疼痛、消化 不良、胃灼热和与过量胃酸相关的症状 ${ }^{[28]}$. 近年来, 对于LDHs的生物医学研究主要集中在LDHs及其插层 结构纳米异质材料在药物输送和治疗等方面的应用.

引用格式： 梅旭安,彭刘琪, 梁瑞政, 卫敏. LDHs生物医学复合材料的制备及其在药物输送和诊疗方面的应用. 中国科学: 化学, 2017, 47: 431-441 Mei X, Peng L, Liang R, Wei M. The fabrication of layered double hydroxides based bio-materials and their application in theranostic and drug delivery. Sci Sin Chim, 2017, 47: 431-441, doi: 10.1360/N032016-00226 
LDHs 可以实现对于生物活性分子如基因、药物和营 养物等活性分子的负载、输送和可控释放 ${ }^{[29-33]}$.

由于LDHs自身带正电, 带负电的生物活性分子 (如核苷、ATP、DNA和维生素)或抗癌药物(如甲氨蝶 呤、5-氟尿嘧啶和阿霉素) 可以通过静电作用插层进 入LDHs层间, 形成Bio-LDHs纳米复合材料 ${ }^{[34-37]}$. LDHs 作为载体可以保护生物活性分子的化学和生物学完整 性, 免受温度、光、水分、 $\mathrm{pH}$ 、酶等参数的影响. 并 进一步减少LDHs中的阴离子型生物分子和带负电荷 的细胞膜之间的静电排斥, 使阴离子生物分子更容易 渗透到细胞内, 从而提高细胞转染效率 ${ }^{[38]}$. 由于 $\mathrm{LDHs}$ 在中性和碱性环境中可以很好地保持其结构, 但在酸 性条件下可溶, 因此, 可以通过将LDHs溶解在弱酸性 细胞质中来释放层间的药物或生物分子, 实现治疗的 目的 ${ }^{[39-41]}$. 在这方面, 最具开创性的工作是通过LDHs 载体将DNA输送到癌细胞中 ${ }^{[42]}$. 由于肿瘤细胞细胞质 $\mathrm{pH}$ 小于 5 , 当DNA-LDHs纳米复合物渗透到肿瘤细胞 后, 原本稳定在LDHs中的DNA分子, 由于LDHs纳米 颗粒的溶解而缓慢释放. 该工作第一次将LDHs应用 于基因疗法, 引起了人们的广泛兴趣. 因此, LDHs可 以作为新型非病毒载体, 高效地保存和输运治疗基因 或药物到人体中的特定靶向部位, 特别是肿瘤细胞中, 达到治疗的目的.

除了治疗应用之外, Bio-LDHs纳米复合材料也应 用于造影剂领域, 以实现成像和诊断功能的强化, 如荧
光成像、光学成像、磁共振成像(MRI)和 X射线计算 机断层扫描成像 $(\mathrm{CT})^{[43-45]}$. 通过对 $\mathrm{LDHs}$ 金属组成及层 间客体的调控, 实现成像诊断功能, 主要可以分为 3 类: (1) 基于被动靶向以及渗透性和保留效应增强(EPR)的 成像; (2) 基于受体介导的主动靶向药物输运和成像; (3) 调控LDHs层板中的金属离子, 使其具有光学成像 或磁共振成像, 并且进一步在层间插入药物或基因分 子形成药物/基因-LDHs, 从而实现沴疗一体化.

本综述重点阐述了多功能Bio-LDHs纳米复合材 料的合成方法、结构特征、化学键合性质和表面修 饰特性(图 1) ${ }^{[41,46-48]}$. 通过细胞及活体实验, 探讨了具 有被动靶向和主动靶向功能的Bio-LDHs体系, 同时 对Bio-LDHs复合材料的成像性能、药物输送治疗、 药代动力学以及生物分布进行了综述和讨论, 并指出 Bio-LDHs复合材料的优势与不足, 展望其发展趋势.

\section{Bio-LDHs的设计}

\section{1 插层法制备Bio-LDHs}

近年来, 由于LDHs独特的二维层状结构及层间和 表面特性, 基于其的插层复合材料受到广泛关注. 利 用插层组装的方法, 赋予了复合材料新的性能或者使 其性能得到极大提升, 为发展LDHs的新型生物无机纳 米复合材料提供了广阔的前景. 由于LDHs自身具有 很高的离子交换特性, 不同种类带负电的生物分子都 (a)

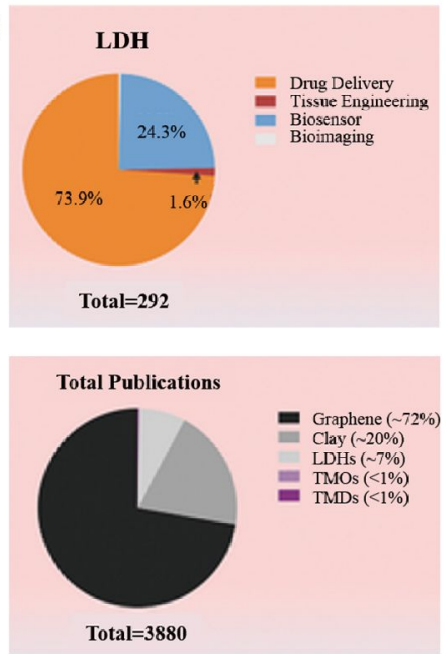

(b)

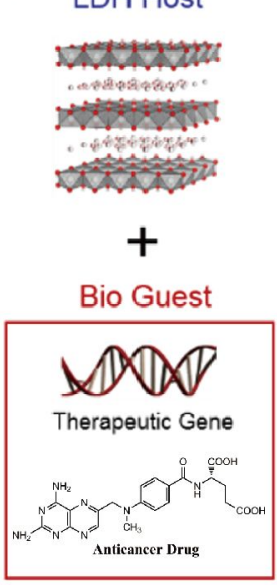

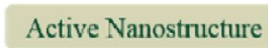

Theranostics
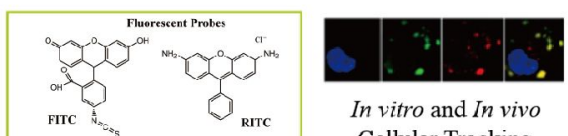

In vitro and In vivo Cellular Tracking

Molecular Engineering
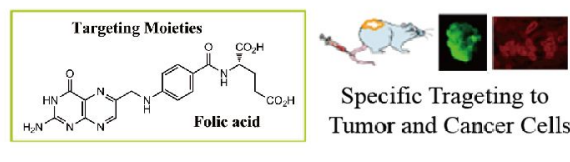

Specific Trageting to Tumor and Cancer Cells

Contrast Reagents

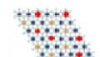

Substitution

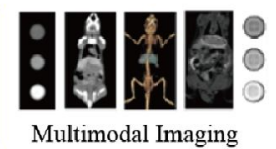

图 1 (a) LDHs在生物医药领域的不同研究方向以及其在二维材料中所占比例示意图 ${ }^{[46]}$; (b) LDHs生物医学复合材料的材 料设计及应用领域 ${ }^{[4,47,48]}$ (网络版彩图) 
能够固定在LDHs层间. 目前, 制备LDHs新型生物无 机纳米复合材料的常见方法包括共沉淀法、离子交 换法、㷽烧复原法和剥层重组法(图2) ${ }^{[49]}$. 利用共沉淀 法制备Bio-LDHs插层复合材料经济可行, 通常方法是 将碱溶液和含有生物分子的金属盐前驱体溶液搅拌 共混并进一步重结晶, 即可制备得到Bio-LDHs插层复 合材料. 离子交换法是制备Bio-LDHs插层复合材料的 另外一种方法, 其中生物分子能否进入到LDHs层间 的决定因素有生物分子的电荷密度、分子大小、层 板金属种类和电荷密度等. 此外, 基于 LDHs的结构记 忆效应, 通过在 $400 \sim 500^{\circ} \mathrm{C}$ 下㷽烧LDHs形成混合金属 氧化物(MMO), 再进一步水合复原, 也能够将一些生 物分子插层到LDHs层间. 而剥层重组法适用于将大 尺寸生物分子插层到LDHs层间, 通过剥层, 将LDHs剥 层成为单层纳米片, 然后将带正电的LDHs纳米片与 带负电的生物分子共同搅拌, 即可进一步将生物分子 插层到LDHs层间.

\subsection{Bio-LDHs 的结构设计及调控}

科研工作者还尝试将Bio-LDHs纳米复合材料制备 成不同的结构和形貌, 如核壳结构、中空结构和介孔 结构. 这些结构特性赋予了Bio-LDHs更加优异的功能. Choy课题组 ${ }^{[2,50]}$ 通过两种反应路径制备DNA-LDHs生

(a) lon-Exchange

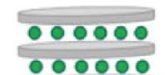

000000

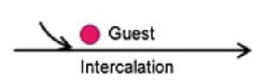

000000

(b) Co-precipitation
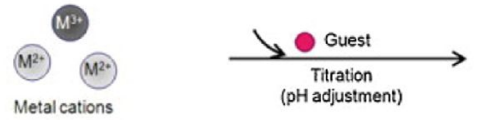

00000

ore्0

(c) Calcination-Reconstruction

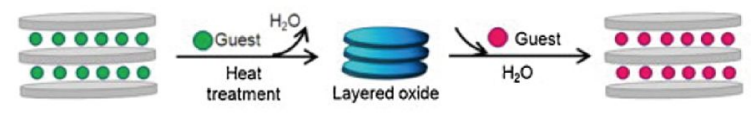

(d) Exfoliation-Reassembling

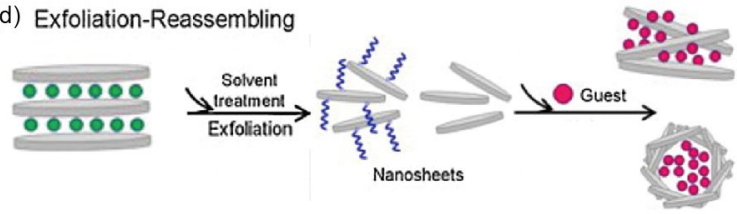

图 2 (a) 离子交换法; (b) 共沉淀法; (c) 㷽烧复原法; (d) 剥 层重组法 ${ }^{[49]}$ (网络版彩图)
物复合材料并得到不同的结构. 一种方法是离子交换 法(图3(a, b)), 通过离子交换法, DNA能够进入到LDHs 的层间, X射线粉末衍射(XRD) 显示LDHs的层间距从 $0.8 \mathrm{~nm}$ 扩展到 $2.4 \mathrm{~nm}$, 扣除 $\mathrm{LDHs}$ 层板厚度 $0.4 \mathrm{~nm}$, 则与 DNA的高度 $(\sim 2 \mathrm{~nm})$ 相符. 同时实验也发现, 螺旋DNA 分子在插层到LDHs层间的过程中会发生一定的扭曲 以适应LDHs层间排布, 动力学模拟也进一步验证了 这种实验现象. 另一种方法是剥层重组法(图3(c)). 通 过将LDHs剥层之后, 再将DNA和LDHs纳米片自组装, 得到核壳结构的DNA-LDHs复合材料. XRD显示, 核 壳结构的DNA-LDHs 具有一个明显的宽峰, 说明它是 无定形的结构. 形貌表征也显示复合材料具有球状 核壳结构, 其中核的粒径在 $100 \mathrm{~nm}$, 壳层厚度为 $10 \mathrm{~nm}$. 球状结构的形成归结于LDHs纳米片围绕DNA分子的 多层自组装. 这样的结构也赋予了DNA分子良好的 物理化学和生物稳定性, 在药物输送、生物医学诊 断和生物信息学等领域具有潜在的应用前景. 本课 题组 ${ }^{[51]}$ 前期通过在 $\mathrm{Fe}_{3} \mathrm{O}_{4}$ 小球上生长 $\mathrm{NiAl}-\mathrm{LDHs}$, 形成 $\mathrm{Fe}_{3} \mathrm{O}_{4} @ \mathrm{SiO}_{2} @ \mathrm{NiAl}-\mathrm{LDHs}$ 花状微球, 该材料具有大的 比表面积和磁学性能. 此外, 由于 $\mathrm{Ni}^{+}$对组氨酸具有选 择性识别和吸附性能, $\mathrm{Fe}_{3} \mathrm{O}_{4} @ \mathrm{SiO}_{2} @ \mathrm{NiAl}-\mathrm{LDHs}$ 复合 材料对于组氨酸标定的绿色荧光蛋白具有吸附分离 性能. 这对于蛋白的纯化和重组具有潜在的运用.

\section{3 多功能Bio-LDHs的分子工程学}

\section{1 具有靶向的Bio-LDHs纳米复合材料}

在肿瘤治疗领域中, 靶向治疗是提高治疗效果、 降低毒副作用的有效途径, 根据不同原理, 靶向治疗 可以分为被动靶向和主动靶向. 被动靶向是指药物在 输送过程中, 实体瘤周围的血管对纳米复合药物具有 高通透性和显著滞留效应. 此外, 当药物通过口服或 注射的方式进入体内后, 血液环境和渗透压决定了药 物在组织器官的分布. 因此, 对于无机纳米载体, 其粒 径、表面电荷等理化性质对其被动靶向的实现有着重 要的作用. 据文献报道(图4(A)), 当水滑石形成均一稳 定的胶体溶液, 并且粒径分布在80 200 nm时, 它将更 易在肿瘤部位富集 ${ }^{[52]}$. Choy课题组 ${ }^{[2]}$ 成功制备了粒径 均一的甲氨蝶呤(MTX)插层LDHs (MTX-LDHs), 其粒 径为 $100 \mathrm{~nm}$, 层间距为 $2.1 \mathrm{~nm}$, 并具有良好的 $\mathrm{LDHs}$ 晶 体结构. 在细胞实验中, MTX-LDHs表现出优异的肿 
(a)

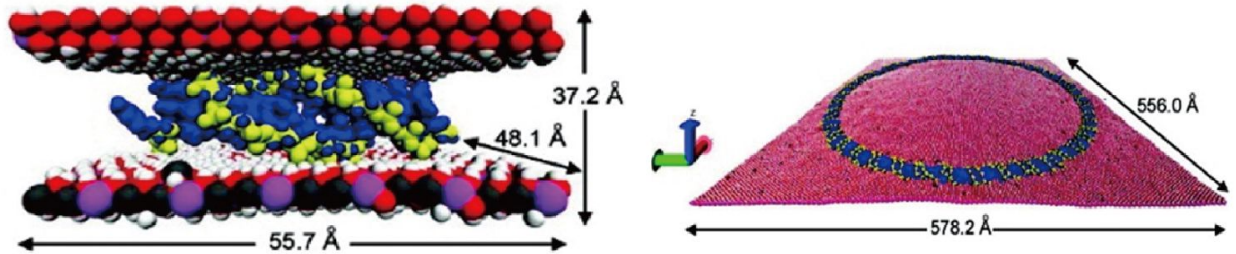

(b)
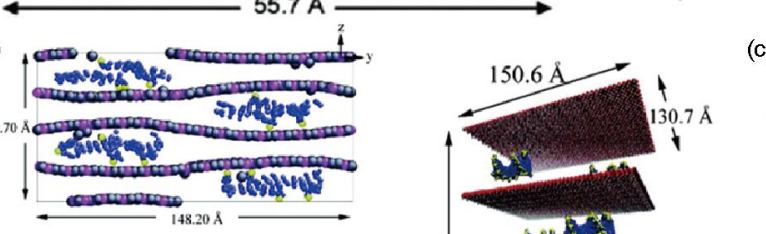

(c) Inorganic
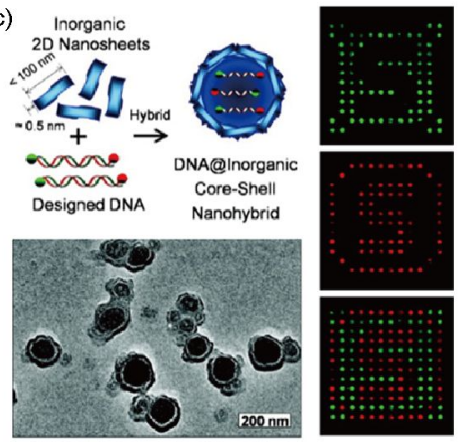

图 $3(\mathrm{a}, \mathrm{b})$ 离子交换法制备DNA-LDHs 复合物及模型构建 ${ }^{[42]}$; (c) 核壳结构的DNA-LDHs 复合材料 ${ }^{[50]}$ (网络版彩图)

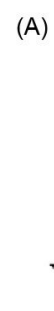

(C)

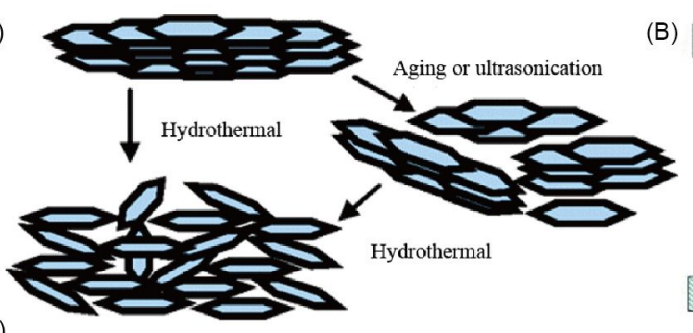

(a)

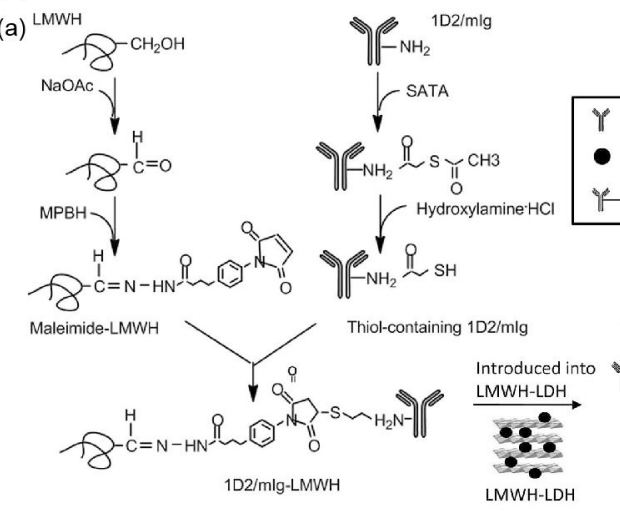

(B)
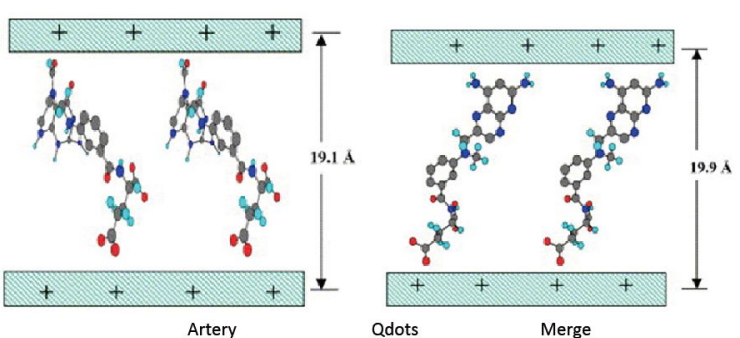

Qdots
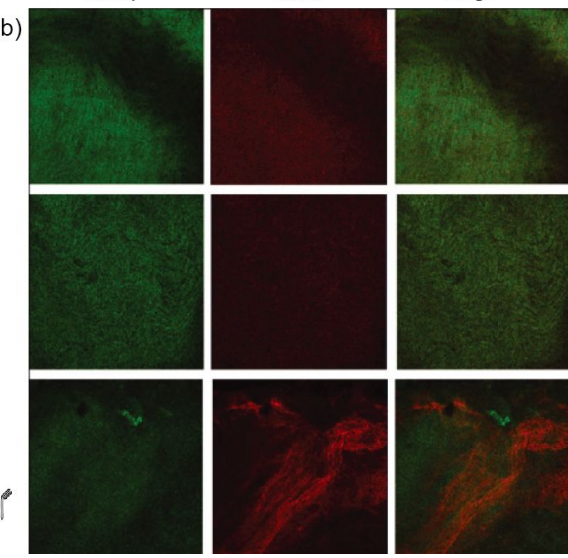

图 4 (A) LDHs粒径及分散性控制示意图; (B) FA及MTX插层LDHs模型 ${ }^{[21]}$; (C) LMWH插层LDHs原理示意图(a)及其苂光效 果图(b) ${ }^{[18]}$ (网络版彩图)

瘤治疗效果, 其 $\mathrm{IC}_{50}$ 相较单一 $\mathrm{MTX}$ 有明显降低. 他们 还通过小鼠实验进一步研究发现MTX-LDHs具有较低 的毒副作用. 在生物环境下, LDHs的胶体稳定性主要 通过表面包覆亲水性基团来实现. 聚乙二醇 $(P E G)$ 是 常用的包覆剂之一, Yan课题组 ${ }^{[53]}$ 报道了聚乙二醇磷
脂包覆的LDHs (PEG-PLDHs), 并且负载药物MTX, 其 粒径在 $130 \mathrm{~nm}$ 左右. 该研究表明, 聚乙二醇磷脂以脂 质体的形式包裹LDHs后, 显著改善了其在血液中的 循环时间, 提高了被动靶向输运能力. 小鼠药代动力 学实验进一步揭示了 PEG-PLDHs-MTX的优异靶向性 
能, 为实际应用提供了依据.

主动靶向是依靠配体和受体的相互识别来实现 的. 为了使水滑石纳米复合物具有主动靶向性能, 基 本策略是选取合适配体与LDHs结合, 利用配体对肿 瘤细胞上受体的识别作用, 这些配体通常包括核酸 适配体、多肽和单克隆抗体等. 目前最常用手段是 利用硅烷偶联反应, 改善水滑石表面物理化学性质. Park 课题组 ${ }^{[54]}$ 首次使用氨丙基三乙氧基硅烷(APS)包 覆 $\mathrm{ZnCr}-\mathrm{LDHs}$, 具体方法是先制备十二烷基硫酸钠插 层的LDHs, 然后加入澳化十六烷基三甲铵(CTAB), 最 终在有机极性溶剂中引入硅烷. Oh课题组 ${ }^{[5]}$ 成功制备 了烷基修饰的LDHs, 并负载药物MTX与靶向分子叶 酸(FA). 在对具有叶酸受体的KB细胞实验中, 由于FA 的主动靶向作用, MTX-LDHs/FA纳米复合物表现出 优异的肿瘤抑制性能(图4(B)). Thomas课题组 ${ }^{[18]}$ 首先 将低分子肝素(LMWH)与单克隆抗体(H93.7C.1D2/48; 1D2)结合, 然后通过静电相互作用将其吸附于LDHs 表面或层间. 该复合物能够有效地输运到血栓部位, 并且具有一定的缓释效果, 在抗血栓及动脉䋆样硬化 方面有一定的应用价值(图4(C)).

\section{2 具有成像功能的Bio-LDHs纳米复合材料}

相较传统材料, 纳米材料在荧光、光学、磁学等 领域具有其独特的性质, 因此被广泛应用于分子成像 领域, 并表现出显影增强、信号放大等优势. LDHs因 其层板阳离子及层间阴离子可调等特性, 在疾病诊断 和肿瘤成像方面具有潜在的应用前景. 目前文献报道 的基于LDHs的成像剂主要由以下4种方法制备: (1) 将 有机染料或稀土配合物与LDHs结合; (2) 用放射性元 素或稀土金属元素替换LDHs层板金属阳离子; (3) 利 用自组装的方法, 使金属或金属氧化物与LDHs结合; (4) 将光功能客体材料与水滑石进行结合.

Choy课题组 ${ }^{[35,47]}$ 选取有机染料异硫氧酸苂光素 (FITC) 以及异硫氧酸罗丹明B (RITC)与MgAl-LDHs结 合, 应用于细胞成像, 并揭示了笼形蛋白介导内吞作 用对无机纳米材料在细胞成像方面的促进作用. 他 们还利用两种不同尺寸的LDHs, 进一步研究了其在 细胞内的输运途径, 并总结了尺寸效应在LDHs作为 药物载体时的关键作用. Rocha课题组 ${ }^{[56]}$ 将 $\mathrm{Eu}^{3+}$ 和 $\mathrm{Nd}^{3+}$ 共同掺杂到LDHs中, 提升了LDHs对可见光、近红外 光的吸收能力, 其展现出优异的近红外发光性能, 结 合其优异的组织穿透能力, 有望进一步应用于生物
成像体系. Chen课题组 ${ }^{[57]}$ 利用原位生长技术, 将5-氟 尿嘧啶插层的LDHs 与 $\mathrm{Y}_{2} \mathrm{O}_{3}: \mathrm{Er}^{3+}, \mathrm{Yb}^{3+}$ 进行结合, 形成 $\mathrm{Y}_{2} \mathrm{O}_{3}: \mathrm{Er}^{3+}, \mathrm{Yb}^{3+} @ \mathrm{SiO}_{2} @ L D H s-5 \mathrm{FU}$ 复合物. 该复合物具 有近红外上转换发光性能, 在 $980 \mathrm{~nm}$ 的激光激发下, 有 很强的红色苂光, 细胞实验也验证了其优异的抗肿瘤 性能. 此类LDHs复合物在诊疗一体化领域展现出潜 在的应用价值. 本课题组 ${ }^{[58]}$ 将金纳米簇针定到剥层水 滑石上, 获得了具备高亮度和稳定性的Au NCs/ELDHs 纳米复合物 (图 5(A, B)). 利用 MgAl-ELDHs 的限域效 应, 金纳米簇的电子转移被抑制, 空穴电子对的复合 得到增强, 实现了金纳米簇量子产率的显著提高. 通 过细胞实验还发现, 该复合物在细胞微环境中仍然 保持良好的稳定性, 并展现出优异的细胞成像性能 (图5(C, D)).

\section{4 诊疗一体化的Bio-LDHs纳米复合材料}

\subsection{Gene-LDHs的应用}

$\mathrm{LDHs}$ 纳米片作为纳米载体, 适用于基因药物的 输运. Choy课题组 ${ }^{[59]}$ 制备了As-myc-LDHs复合物并实 现基因的输运. 通过将反义寡核苷酸As-myc插层到 LDHs中, 有效地将其输运到HL-60肿瘤细胞中, 实现 对肿瘤细胞增殖的有效抑制(图6(A)). Giannelis课题 组 ${ }^{\left[{ }^{[0]}\right.}$ 报道了将绿色苂光蛋白GFP插层到LDHs中, 并且 实现在不同肿瘤细胞中的输运和表达, 其对基因的转 染效率都在 $90 \%$ 以上. Lu课题组 ${ }^{[61]}$ 将异硫氰酸荧光素 负载到超小粒径 $(20 \mathrm{~nm})$ 的 $\mathrm{LDHs}$ 上. 细胞摄取实验表 明, 其具有潜在的基因输送能力, 且随着FITC-LDHs浓 度的增加, 细胞核和细胞质的摄取量线性增加, 显示出 FITC-LDHs在细胞核内良好的输送能力. 透射电镜和 共聚焦显微镜表征显示, 小粒径的LDHs能够进入到细 胞质并大量富集在细胞核中, 而大于 $20 \mathrm{~nm}$ 的LDHs 只 能富集在细胞质, 不能进入细胞核中(图6(B)). 因此将 此LDHs纳米片进一步负载质粒DNA (pEGFP-N1), 可 成功实现对NSC 34细胞转染及对绿色荧光蛋白的表 达. Xu课题组 ${ }^{[62]}$ 也报道了小且规则的LDHs纳米片能 够有效地将干扰核糖核酸分子(siRNA)输运到肿瘤细 胞细胞质中, 实现对信使RNA的降解. 此外, 他们也报 道了siRNA和抗肿瘤药物 5-氟尿嘧啶 (5-FU)共同负载 到LDHs上, 可以实现对肿瘤的协同治疗 ${ }^{[63]}$. 通过网格 蛋白介导的内吞作用,复合材料能够很好地进入到细 

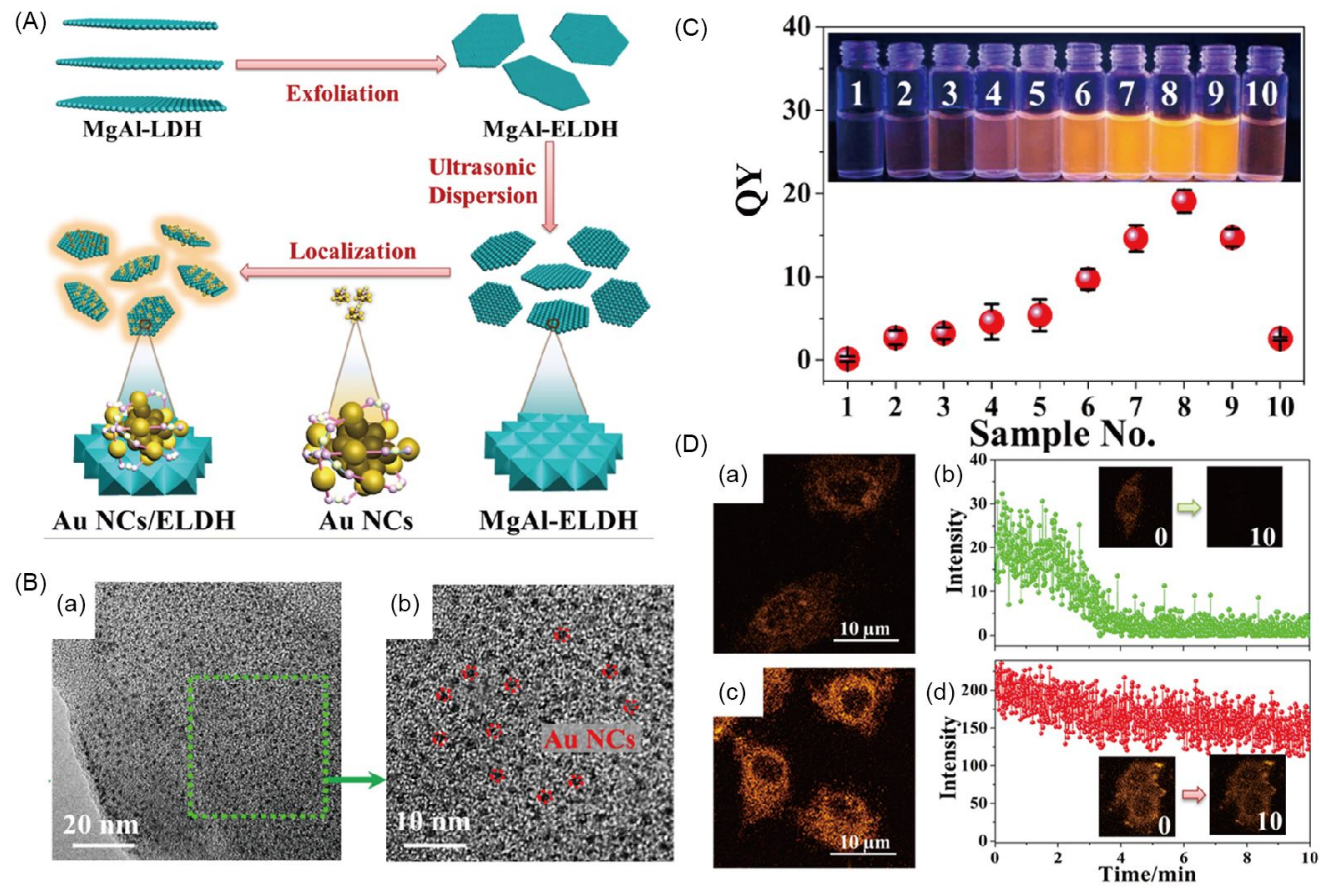

图 $5 \mathrm{AuNCs} / \mathrm{ELDHs}$ 的(A)制备示意图、(B) HRTEM照片、(C)量子产率及苂光照片、(D)细胞成像(a, c) 及时间稳定性(b, d) ${ }^{[58]}$ (网络版彩图)

胞质中, 并且显示出比单一治疗更优异的抗肿瘤性 能(图6(C)). 此外, 将药物分子和成像分子共同负载到 LDHs上, 实现成像和治疗的协同, 对于实现高效的诊 疗性能具有显著的促进作用. Choy课题组 ${ }^{[48]}$ 开发了一 种 siRNA-LDHs 诊疗复合材料, 可实现靶向肿瘤治疗 和成像. 主要方法是通过将siRNA、FITC和叶酸(FA), 共同负载在 LDHs上, 赋予该纳米材料选择性的肿瘤 靶向能力. 实验表明, 其细胞摄取量相较于没有叶酸 的LDHs/FITC复合材料提高了1.5倍(图6(D)). 活体实 验表明, 腹腔注射 siRNA-LDHs/FA后, 通过干预肿瘤细 胞mRNA的表达, 肿瘤的增殖受到明显抑制, 其性能也 明显强于 siRNA-LDHs.

\subsection{Drug-LDHs 的应用}

除了对于基因的输送外, 各类活性药物, 包括抗肿 瘤药物、消炎药物以及心血管药物通过负载到LDHs 上, 也能够实现良好的药物缓释, 延长药物的半衰期, 增加药物富集以及增强药效. Zhu课题组 ${ }^{[64]}$ 报道了一 类新型的顺铂负载LDHs的抗肿瘤药物, 其具有优异的 抗肿瘤性能. 将顺铂药物插层到LDHs层间能够降低 顺铂对正常细胞的细胞毒性. 同时, 复合物能够被肿
瘤细胞高效摄取, 通过与DNA结合抑制细胞增殖, 实 现肿瘤治疗. Li课题组 ${ }^{[65]}$ 制备了一种具有多种功能的 LDHs核壳结构, 通过在磁性纳米微球表面生长LDHs, 并且负载抗肿瘤药物DOX和荧光成像剂FITC, 成功实 现成像和治疗一体化. 他们发现, 肿瘤细胞酸性的细 胞质是增加药物释放的关键因素. 在酸性细胞质中, DOX-LDHs的酰胺键能够被有效破环, 因此能够实现 DOX的快速释放. 这个现象只发现在肿瘤细胞中, 而 在正常细胞中没有, 说明复合材料具有选择性释放的 能力, 这对于增加药物药效, 同时降低药物毒性非常 关键. Shi课题组 ${ }^{[41]}$ 同样制备了一类磁共振和 CT双模 成像的诊疗药物. 通过在LDHs层板掺杂 $\mathrm{Gd}$ 元素, 进一 步负载金纳米颗粒和抗肿瘤药物 DOX, 成功实现成像 和治疗. DOX负载到LDHs后通过内吞进入到肿瘤细 胞, 在酸性细胞质中, 其释放效果得到显著增强, 因此 其抗肿瘤性能相较于DOX本身也有了显著增强. 此 外, 复合物相较于商用的磁成像剂及 $\mathrm{CT}$ 成像剂, 其成 像性能也得到了显著增强.

此外, LDHs还能够用于对各类光热试剂的负载. 光学治疗是一种引入光活性物质与肿瘤细胞相结合, 通过外加光照激发, 实现肿瘤细胞坏死凋亡的治疗方 


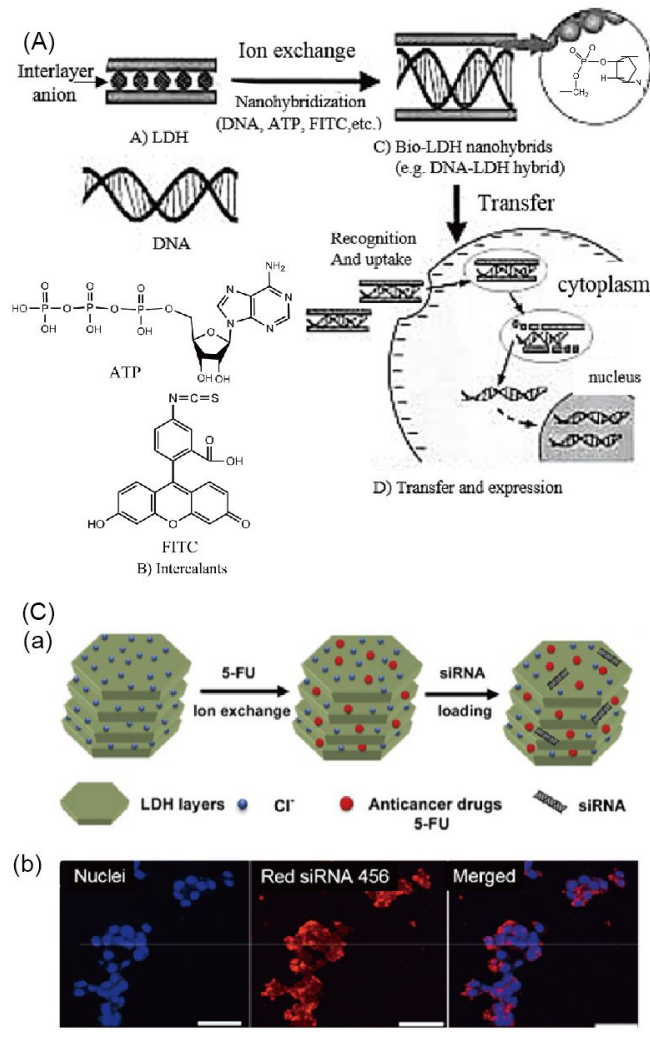

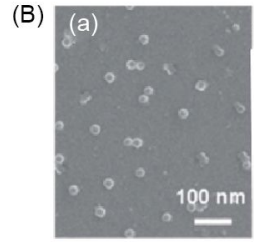
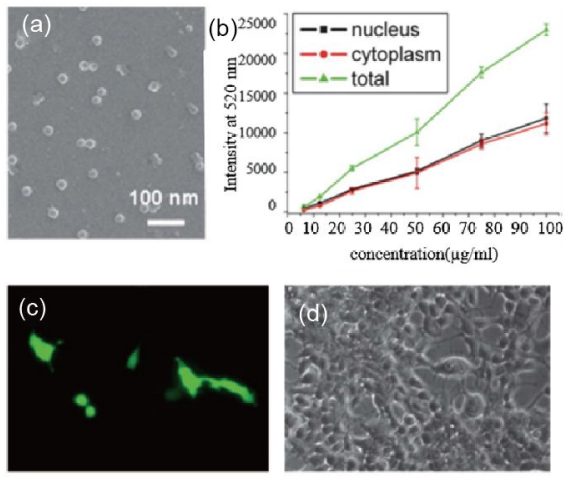

(D)
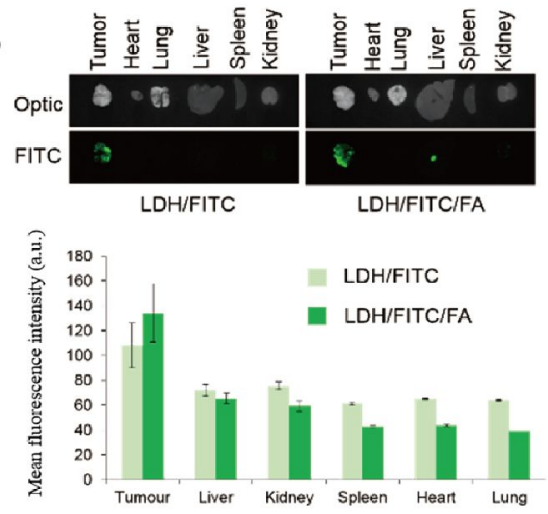

图 6 (A) As-myc-LDHs复合物制备原理图 ${ }^{[59]}$; (B) 超小粒径FITC-LDHs电镜及共聚焦图 ${ }^{\left[{ }^{[61]}\right.}$; (C) siRNA和抗肿瘤药物 5-FU共 同负载LDHs制备原理(a)及苂光成像图(b) ${ }^{[63]}$; (D) siRNA-LDHs/FA诊疗复合材料在不同器官的累积量 ${ }^{[48]}$ (网络版彩图)

法. 其具有副作用小、可协同治疗、可控治疗的优势. 光敏剂和光热试剂是决定光动力治疗和光热治疗的 关键因素. 单一光敏剂在应用过程中由于苯环的 $\pi-\pi$ 堆积会导致二聚体及多聚体的产生, 聚集体的存在使 得光敏剂从激发态过渡到三线态的能力降低, 因此生 成单态氧的能力大大降低. 本课题组 ${ }^{[66]}$ 通过将酞菁锌 插层到水滑石层间实现了抑制酞菁锌的聚集. 酞菁锌 /LDHs复合光敏剂生成单态氧的能力比起酞菁锌自身 得到极大的提高. 细胞实验表明, 在极低的药物使用 量上 $(10 \mu \mathrm{g} / \mathrm{mL})$ 能够实现对 $85.7 \%$ 的肿瘤细胞的抑制 效果. 小鼠的体内实验也证明, 酞菁锌/LDHs复合光敏 剂在极低的药物用量 $(0.3 \mathrm{mg} / \mathrm{kg})$ 及较低的光通量 $(54$ $\mathrm{J} / \mathrm{cm}^{2}$ ) 照射下对于活体肿瘤有良好的抑制作用(图7). 同时, 酞菁锌/LDHs复合光敏剂相较于酞菁锌具有更 好的光稳定性和生物相容性. 本课题组 ${ }^{[67]}$ 进一步采 用共沉淀方法将有机光热试剂吲哚菁绿(ICG) 和靶向 分子叶酸(FA)共插层进 $\mathrm{LDHs}$ 层间构建复合光热试剂
ICG-FA/LDHs. 由于主体-客体和客体-客体的相互作 用, ICG 在 LDHs层间以单分子态存在, 从而导致复合 材料的光热转换效率与未插层ICG相比提升了 $11.4 \%$. 细胞实验表明, 具有叶酸受体的癌细胞 (KB细胞)对复 合材料吸收较好, 表现出近红外成像性能. 在光热治 疗效果测试中, 较低浓度的复合材料(ICG 当量浓度 8 $\mu \mathrm{g} / \mathrm{mL})$ 在较低能量的近红外光照条件下 $\left(1.1 \mathrm{~W} / \mathrm{cm}^{2}, 12\right.$ $\mathrm{min})$, 对 KB细胞达到 $87.4 \%$ 的细胞杀伤力. 此外, 复合 光热试剂还表现出良好的生物相容性和存储稳定性, 具有潜在的应用前景.

\section{5 结论}

本文详细介绍了Bio-LDHs复合材料的材料设计、 制备方法、结构控制及在诊疗和药物输送方面的应 用. 同时也介绍了Bio-LDHs复合材料在主动和被动靶 向诊疗方面的最新进展. 通过对LDHs进行靶向设计 和修饰, 药物分子和成像剂都能很好地与病变组织结 
(A)

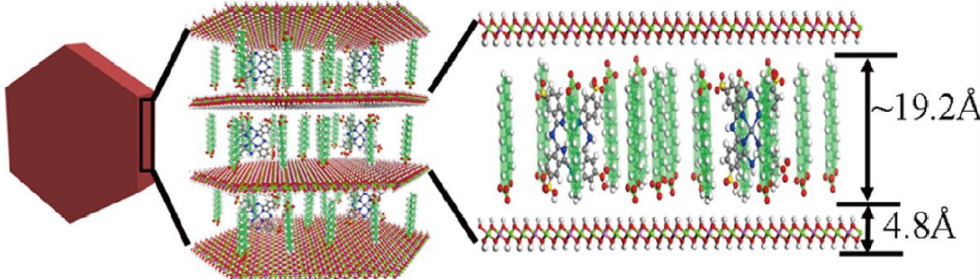

(B)
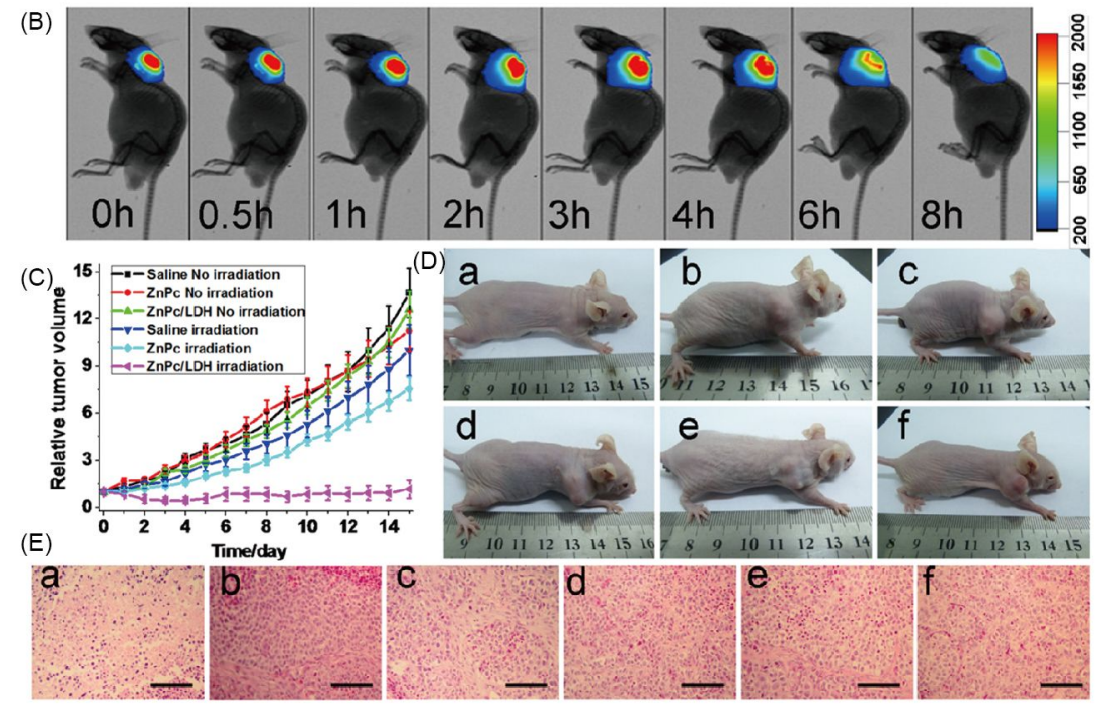

图 7 酞菁锌/LDHs复合光敏剂的(A)结构模拟图、(B)小鼠活体成像、(C)小鼠肿瘤体积在不同时间点的变化曲线、(D) 14 $\mathrm{d}$ 后小鼠肿瘤的照片和(E)小鼠肿瘤部位切片 ${ }^{[66]}$ (网络版彩图)

合, 达到诊断和治疗的目的, 这在临床医学上具有潜 在的应用前景. 但是, 实现Bio-LDHs纳米复合材料在 临床医学领域的应用, 还需要克服诸多困难, 主要包 括以下 5 个方面: (1) 如何普遍增强Bio-LDHs纳米复合 材料的稳定性. 单一 LDHs具有良好的稳定性, 但是当 LDHs 与生物活性分子相结合时会显著降低其胶体稳 定性, 这不利于Bio-LDHs纳米复合材料在体内的输送 和摄取, 还会造成血管堵塞、代谢困难和药效低下等 副作用. (2) 如何显著降低生物活性分子特别是药物 分子的副作用. 化疗药物普遍具有较大的毒性, 将其 插层到LDHs层间后, 通过被动靶向及选择性释放可以 部分降低其细胞毒性, 但是效果并不显著. (3) LDHs作 为药物载体, 自身并不具备其他功效, 因此功能相对单 一, 相较于其他无机纳米载体, 不能体现明显优势. 此 外, 药物负载到 $\mathrm{LDHs}$ 上, 其药效和毒性并未表现出显 著的优势. (4) 如何降低正常组织和器官对Bio-LDHs 纳米复合材料的排斥性. 由于Bio-LDHs纳米复合材料 属于外源性材料, 进入到组织和器官后很容易引起排 斥性和副反应, 这也会显著影响患者的各项生理指标.
(5) 目前针对Bio-LDHs纳米复合材料, 只是从细胞和 动物活体层次进行考察, 还未在临床上进行验证, 其 在人体内的输送、药效、毒理性质、药物沉积和代 谢等都缺乏进一步的探索. 这对于Bio-LDHs纳米复合 材料的发展提出了很大挑战.

针对以上问题, 相应的解决途径主要表现在以下 5 个方向: (1) 针对Bio-LDHs稳定性的问题, 可以选择生 物相容性好的材料对Bio-LDHs纳米复合材料进行二 次修饰. 例如, 通过进一步硅烷化或采用聚合物包覆 增强其稳定性. (2) 针对Bio-LDHs如何降低药物分子 副作用的问题,一个方面可以通过跟抗体等具有专一 性、特异性靶向材料结合, 增强其对病变组织的靶向 输送和摄取; 另一个方面, 通过结构设计引入具有刺 激响应释放的功能来实现选择性的药物释放, 如 $\mathrm{pH}$ 、 温度、光照、超声、酶等刺激性响应释放. (3) 针对 LDHs自身功能相对单一的问题, 可以通过层板金属组 成调控、结构调控、层间客体插层, 表面进一步修改 等手段, 赋予LDHs自身的多功能化, 目前被选用作为 药物载体的LDHs 都是最常见的LDHs, 如MgAl-LDHs 
和ZnAl-LDHs等. 通过进一步研究其他金属类型的 LDHs作为药物载体的性能, 不仅能够增加药物载体的 LDHs种类, 而且还可以赋予 LDHs载体自身的其他功 能. (4) 针对正常组织和器官对Bio-LDHs纳米复合材 料的排斥性和生物相容性的问题, 可以引入一些内源 性物质进行进一步修饰, 降低组织和器官对Bio-LDHs 纳米复合材料的排斥性. 例如, 在复合物表面进一步 修饰上一些生物相容性好的磷脂等内源性材料, 增强 生物相容性和摄取能力. (5) 针对Bio-LDHs纳米复合
材料从基础研究过渡到临床研究的问题, 可以分两个 方向来发展, 一是加强与医疗机构的合作, 将目前比 较成熟的成果推广到临床研究; 另一个是加强基础研 究, 以运用为导向, 切实解决实际运用过程当中的一 些关键科学问题, 并实现其关键性能的显著提升. 总 而言之, 随着科学的不断发展, 基于LDHs的生物医学 复合材料也将得到快速发展. 我们有理由相信, 随着 研究的不断深入, LDHs的生物医学复合材料会真正 进入到临床医学得到实际应用.

\section{参考文献}

1 Whittingham MS. Science, 1976, 192: 1126-1127

2 Sutter P, Sadowski JT, Sutter EA. J Am Chem Soc, 2010, 132: 8175-8179

3 Benavente E, Santa Ana MA, Mendizabal F, Gonzalez G. Coodin Chem Rev, 2002, 224: 87-109

4 Wu X, Cao Y, Ai X, Qian J, Yang H. Electrochem Commun, 2013, 31: 145-148

Sun Y, Cheng H, Gao S, Liu Q, Sun Z, Xiao C, Wu C, Wei S, Xie Y. J Am Chem Soc, 2012, 134: 20294-20297

Wang Z, Zhu W, Qiu Y, Yi X, von dem Bussche A, Kane A, Gao H, Koski K, Hurt R. Chem Soc Rev, 2016, 45: 1750-1780

Liang R, Wei M, Evans DG, Duan X. Chem Commun, 2014, 50: 14071-14081

Tan C, Liu Z, Huang W, Zhang H. Chem Soc Rev, 2015, 44: 2615-2628

9 Wang K, Wang J, Fan J, Lotya M, O'Neill A, Fox D, Feng Y, Zhang X, Jiang B, Zhao Q, Zhang H, Coleman JN, Zhang L, Blau WJ. ACS Nano, 2013, 7: 9260-9267

10 Choy JH, Kwak SY, Park JS, Jeong YJ, Portier J. J Am Chem Soc, 1999, 121: 1399-1400

11 Liu Z, Robinson JT, Sun X, Dai H. J Am Chem Soc, 2008, 130: 10876-10877

12 Chen Y, Ye D, Wu M, Chen H, Zhang L, Shi J, Wang L. Adv Mater, 2014, 26: 7019-7026

13 Liu T, Wang C, Gu X, Gong H, Cheng L, Shi X, Feng L, Sun B, Liu Z. Adv Mater, 2014, 26: 3433-3440

14 Feng L, Wu L, Qu X. Adv Mater, 2013, 25: 168-186

15 Cheng L, Liu J, Gu X, Gong H, Shi X, Liu T, Wang C, Wang X, Liu G, Xing H, Bu W, Sun B, Liu Z. Adv Mater, 2014, 26: 1886-1893

16 Wang H, Yang X, Shao W, Chen S, Xie J, Zhang X, Wang J, Xie Y. J Am Chem Soc, 2015, 137: 11376-11382

17 Thakur A, Jaiswal MK, Peak CW, Carrow JK, Gentry J, Dolatshahi-Pirouz A, Gaharwar AK. Nanoscale, 2016, 8: 12362-12372

18 Gu Z, Rolfe BE, Xu ZP, Campbell JH, Lu GQM, Thomas AC. Adv Healthcare Mater, 2012, 1: 669-673

19 Li X, Zhu J, Wei B. Chem Soc Rev, 2016, 45: 3145-3187

20 Jun H, Zhiliang Z, Hongtao L, Yanling Q. RSC Adv, 2014, 4: 5156-5164

21 Oh JM, Park M, Kim ST, Jung JY, Kang YG, Choy JH. J Phys Chem Solids, 2006, 67: 1024-1027

22 Song F, Hu X. Nat Commun, 2014, 5: 4477-4485

23 Yan Y, Liu Q, Wang J, Wei J, Gao Z, Mann T, Li Z, He Y, Zhang M, Liu L. J Colloid Interface Sci, 2012, 371: 15-19

24 Wang Q, Tay HH, Zhong Z, Luo J, Borgna A. Energy Environ Sci, 2012, 5: 7526

25 Wang Q, Tang Vi SY, Lester E, O'Hare D. Nanoscale, 2013, 5: 114-117

26 Evans DG, Duan X. Chem Commun, 2006, 11: 485-496

27 Williams GR, Fogg AM, Sloan J, Taviot-Guého C, O'Hare D. Dalton Trans, 2007, 29: 3499

28 Funnell NP, Wang Q, Connor L, Tucker MG, O'Hare D, Goodwin AL. Nanoscale, 2014, 6: 8032-8036

29 Li L, Gu W, Liu J, Yan S, Xu ZP. Nano Res, 2015, 8: 682-694

30 Gu Z, Atherton JJ, Xu ZP. Chem Commun, 2015, 51: 3024-3036

31 Kuthati Y, Kankala RK, Lee CH. Appl Clay Sci, 2015, 112-113: 100-116

32 Gu Z, Zuo H, Li L, Wu A, Xu ZP. J Mater Chem B, 2015, 3: 3331-3339

33 Barahuie F, Hussein MZ, Gani SA, Fakurazi S, Zainal Z. J Solid State Chem, 2015, 221: 21-31

34 Choi SJ, Choi GE, Oh JM, Oh YJ, Park MC, Choy JH. J Mater Chem, 2010, 20: 9463-9469 


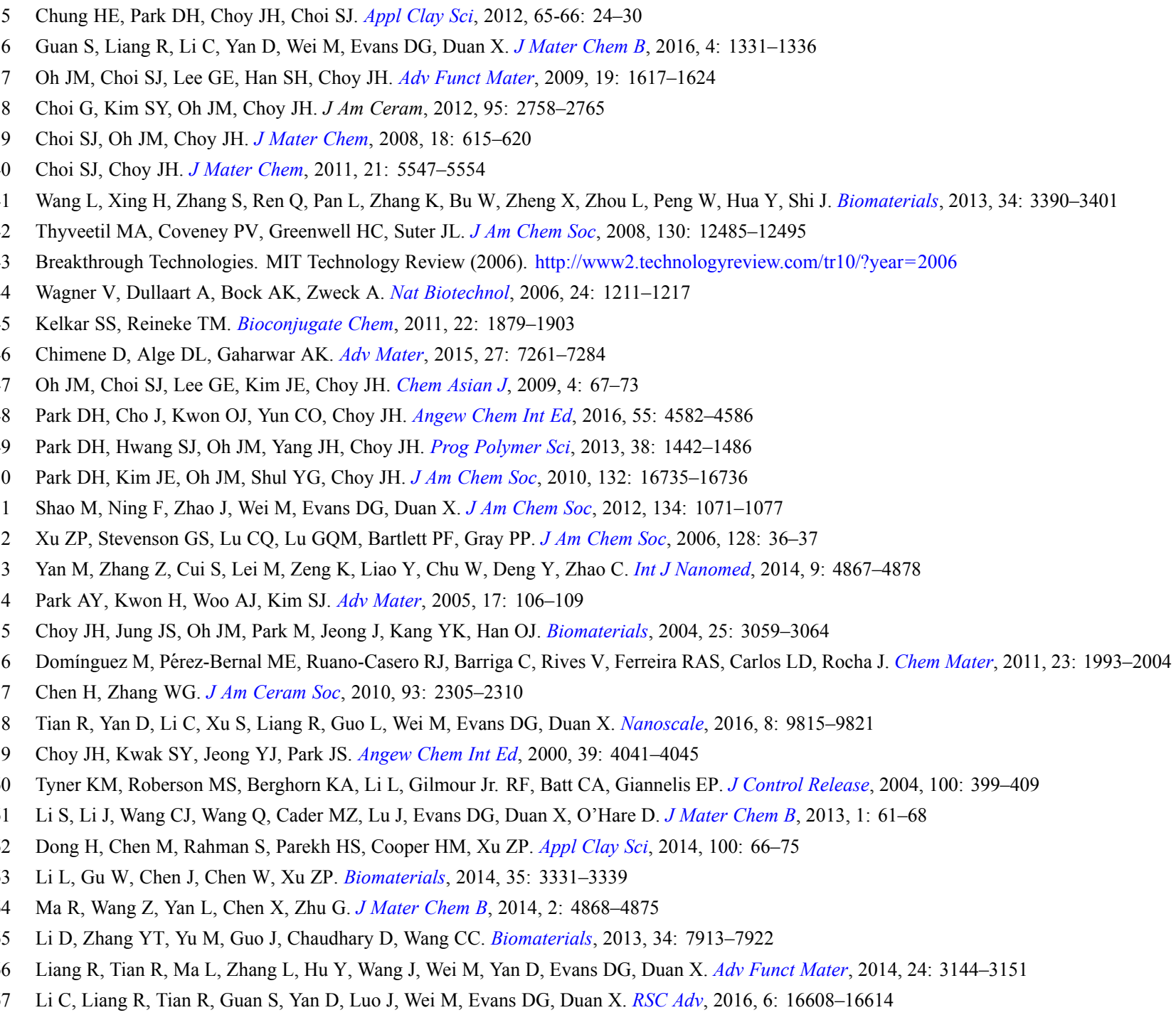




\title{
The fabrication of layered double hydroxides based bio-materials and their application in theranostic and drug delivery
}

\author{
Xu'an Mei, Liuqi Peng, Ruizheng Liang", Min Wei* \\ State Key Laboratory of Chemical Resource Engineering, Beijing University of Chemical Technology, Beijing 100029, China \\ *Corresponding authors (email: liangruizheng2000@163.com; weimin@mail.buct.edu.cn)
}

\begin{abstract}
With the fast development of bio-related science, theranostic composite materials have recently attracted considerable attention due to their advantages in the combination of diagnosis and treatment. Layered double hydroxides (LDHs) have been widely applied as various multi-functional materials and exhibited great potential in the construction of bio-materials. This review introduces the construction of new hybrid materials based on LDHs host and bio-materials through intercalation assembly. Aiming at fabrication of hybrid materials with superior performances in diagnosis and treatment, we expatiate upon the intercalation structure, the arrangement of guests and host-guest interaction to achieve the synergistic effect of LDHs host and intercalated biomaterials. Moreover, the growing trend and promising new strategies are discussed from the point of view of specific clinical challenges.
\end{abstract}

Keywords: LDHs, intercalation, theranostic, drug delivery, bio-inorganic nanomaterials

doi: $10.1360 / \mathrm{N} 032016-00226$ 